\title{
INTERPRETAÇÕES PALEOCLIMÁTICAS A PARTIR DA TAFOFLORA DE CAIEIRA, FORMAÇÃO PIRABAS, OLIGOCENO/MIOCENO DA AMAZÔNIA ORIENTAL, PARÁ, BRASIL
}

\author{
FRANCISCO SANTIAGO \\ Programa de Pós-Graduação em Geociências, Instituto de Geociências, Universidade Estadual de Campinas, Cx.P. 6152, \\ 13083-970, Campinas, SP, Brasil. santiago1403@gmail.com \\ FRESIA RICARDI-BRANCO \\ Departamento de Geologia e Recursos Naturais, Instituto de Geociências, Universidade Estadual de Campinas, Cx.P. 6152, \\ 13083-970, Campinas, SP, Brasil.fresia@ige.unicamp.br
}

\begin{abstract}
Paleoclimatic interpretations from the Caieira taphoflora, Pirabas Formation, Oligocene/Miocene of the eastern Amazonia, Pará, Brazil. The Caieira taphoflora, which is associated with the Pirabas Formation, is the best documented one for the Oligocene/ Miocene of the eastern Amazonia. The taphoflora represents a predecessor of a phytophysiognomy of the Amazon Forest, specifically of a floodplain forest, which has developed under a warm and humid climate. The main physiognomic characteristics - type of margins and leaf area - of the angiosperm leaves of 19 species of this taphoflora, allowed us to carry out the reconstruction of the mean annual temperature (MAT) and the mean annual precipitation (MAP) for the moment of its deposition. The different equations used to reconstruct the MAT and the MAP are based on univariate methods known as Leaf Margin Analysis and Leaf Area Analysis. These equations indicated that the Caieira taphoflora developed under a MAT between 24.6 and $25.0^{\circ} \mathrm{C}$, and a MAP between 1849 and $2423 \mathrm{~mm}$. The values are similar to the current values for the region where the taphoflora was collected, therefore, it is possible to infer that the current climatic configuration has been firmly established since the Oligocene/Miocene, as well as its vegetation. The values also indicate that the climate was colder, between 1.1 and $1.5^{\circ} \mathrm{C}$, and less humid, between 42 and $619 \mathrm{~mm}$, for the moment of deposition of the Caieira taphoflora, possibly due to the global cooling event known as Mi-1 glaciation, which affected the whole Amazonia during the Oligocene/Miocene transition.
\end{abstract}

Keywords: Oligocene/Miocene, eastern Amazonia, warm and humid climate, Leaf Margin Analysis, Leaf Area Analysis, Mi-1 glaciation.

RESUMO - A tafoflora de Caieira, a qual se encontra associada à Formação Pirabas, é a mais bem documentada para o Oligoceno/Mioceno da Amazônia oriental. Esta tafoflora é considerada um predecessor de uma fitofisionomia da Floresta Amazônica, especificamente de uma floresta de planície inundável, o qual se desenvolveu sob um clima quente e úmido. As principais características fisionômicas, tipo de margem e área foliar, das folhas de angiospermas de 19 espécies desta tafoflora, permitiram reconstruir a temperatura média anual (TMA) e a precipitação média anual (PMA) para o momento de sua deposição. As diferentes equações utilizadas para reconstruir a TMA e a PMA estão baseadas nos métodos univariados conhecidos como Análise da Margem Foliar e Análise da Área Foliar. Estas equações indicaram que a tafoflora de Caieira se desenvolveu sob uma TMA de 24,6 a 25,0 $0^{\circ} \mathrm{C}$ e uma PMA de 1849 a $2423 \mathrm{~mm}$. Estes valores, se assemelham aos que apresenta hoje a região onde esta tafoflora foi coletada, portanto, é possível inferir que a atual configuração climática tem estado firmemente estabelecida desde o Oligoceno/Mioceno, assim como sua vegetação. Estes valores também indicam que para o momento de deposição da tafoflora de Caieira o clima era mais frio, em 1,1 a $1,5^{\circ} \mathrm{C}$, e menos úmido, em 42 a $619 \mathrm{~mm}$, possivelmente devido ao evento de arrefecimento global conhecido como glaciação Mi-1, o qual afetou de maneira geral toda a Amazônia durante a transição Oligoceno/Mioceno.

Palavras-chave: Oligoceno/Mioceno, Amazônia oriental, clima quente e úmido, Análise da Margem Foliar, Análise da Área Foliar, glaciação Mi-1.

\section{INTRODUÇÃO}

As plantas, devido à sua abundância, diversidade e distribuição, são valiosas como indicadores climáticos (Wright et al., 2017). A estrutura da planta mais utilizada como indicador climático é a folha, já que esta responde rapidamente às condições ambientais a que está exposta (Royer, 2012). Por esta razão, durante quase um século as folhas das plantas têm sido amplamente empregadas pelos paleobotânicos para reconstruir os climas terrestres do Cretáceo e do Cenozoico (Burnham \& Tonkovich, 2011; Peppe et al., 2011; Royer, 2012; Wright et al., 2017). Devido à sua simplicidade, os métodos mais utilizados para a reconstrução dos principais parâmetros climáticos, temperatura média anual (TMA) e precipitação média anual (PMA), com base nas características fisionômicas das folhas são os conhecidos como Análises 
da Margem Foliar (Wilf, 1997; Kennedy et al., 2014) e Análises da Área Foliar (Wilf et al., 1998). Estes métodos, estão baseadas na relação que existe entre o tipo de margem e a TMA, e entre o tamanho da folha e a PMA (Burnham \& Tonkovich, 2011; Peppe et al., 2011; Royer, 2012).

$\mathrm{Na}$ localidade de Caieira, no nordeste do Estado do Pará, região Norte do Brasil, foi registrada uma flora fóssil, composta exclusivamente por folhas de angiospermas, que representa um predecessor de uma fitofisionomia da Floresta Amazônica (Duarte, 1972, 2004; Rossetti \& Toledo, 2007; Jaramillo et al., 2010). A tafoflora de Caieira se encontra associada a uma sequência da Formação Pirabas (Ackerman, 1964; Duarte, 1972, 2004). Esta formação se depositou em ambientes marinhos costeiros, e está constituída por rochas carbonáticas e siliciclásticas, as quais registram os eventos transgressivos/regressivos mais importantes que ocorreram ao longo da costa da Amazônia oriental durante o Oligoceno/ Mioceno (Rossetti \& Góes, 2004; Hoorn et al., 2010; Aguilera et al., 2014; Antonioli et al., 2015; Silva, 2016).

Uma vez a que as principais características fisionômicas das folhas de angiospermas da tafoflora de Caieira se encontram bem preservadas, o presente trabalho pode objetivar a reconstituição dos principais parâmetros climáticos - TMA e PMA - para o momento de sua deposição, utilizando os métodos conhecidos como Análises da Margem Foliar e a Análises da Área Foliar.

\section{ÁREA DE ESTUDO}

No nordeste do Estado do Pará, a 5,4 km a leste da cidade de Capanema, situa-se a localidade de Caieira (Figura 1), onde foi coletada a tafoflora de Caieira, em uma seção de uma escavação a céu aberto. Esta seção era de aproximadamente $5 \mathrm{~m}$ de espessura (Ackerman, 1964), e se caracterizava pela intercalação de camadas de calcário creme a esbranquiçada e de argilitos calcíferos cinza-escuros, ricos em impressões de folhas (Duarte, 2004). Estas camadas foram depositadas durante uma fase de progradação instalada após a transgressão marinha, quando a borda da bacia soergueu e foi inibida a deposição de carbonatos (Távora et al., 2010a). O ambiente de deposição destas camadas era litorâneo de águas rasas e calmas (Duarte, 2004), especificamente de plataforma restrita/lagunar sob forte influência de processos de marés em contextos de estuário e mangue (Duarte, 2004; Távora et al., 2010a).

O estudo da tafoflora de Caieira permitiu identificar 20 novas espécies, as quais foram inicialmente descritas por Duarte (1972) e posteriormente publicadas por Duarte (2004). Estas espécies se encontram associadas às famílias Annonaceae, Bonnetiaceae, Caryocaraceae, Chrysobalanaceae, Dilleniaceae, Ebenaceae, Euphorbiaceae, Lauraceae, Malvaceae, Melastomataceae, Meliaceae, Myrtaceae, Nyctaginaceae, Rapataceae, Rhizophoraceae, Rubiaceae, Rutaceae e Sapindaceae (Duarte, 1972, 2004). A análise da composição taxonômica da tafoflora de Caieira, assim como das características fisionômicas das folhas - área foliar, ápice e venação - , permitiram inferir que a tafoflora pode representar um predecessor de uma fitofisionomia da Floresta Amazônica, especificamente de uma floresta de planície inundável (Duarte, 1972, 2004), que teria se desenvolvido sob um clima tropical quente e úmido (Duarte, 1972, 2004; Jaramillo et al., 2010; Hoorn et al., 2014).

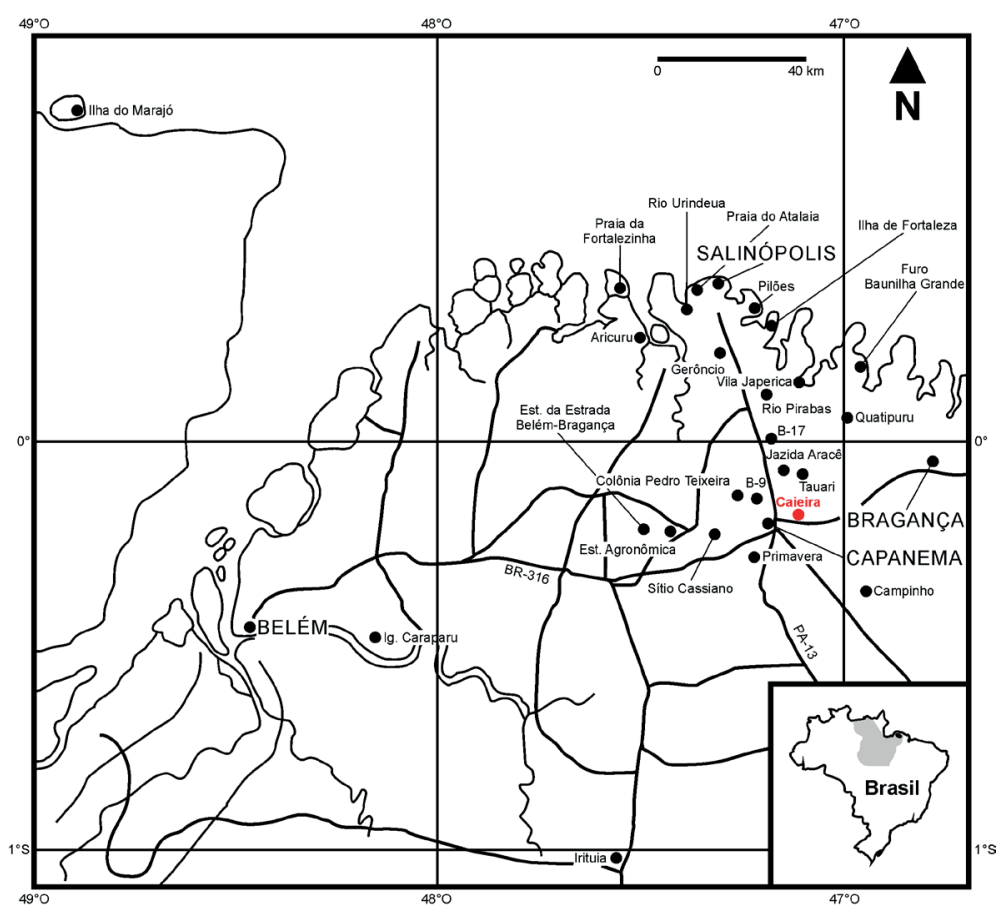

Figura 1. Localidades fossilíferas da Formação Pirabas no Estado do Pará (Távora et al., 2010b).

Figure 1. Fossiliferous localities of the Pirabas Formation in the State of Pará (Távora et al., 2010b). 


\section{MATERIAL E MÉTODOS}

\section{Flora fóssil}

Neste estudo foi analisado o tipo de margem e tamanho foliar de 19 das 20 espécies de angiospermas identificadas para a tafoflora de Caieira (Tabela 1). Os dados foram adquiridos das descrições de sua arquitetura foliar, as quais foram tomadas da bibliografia (Duarte, 1972). Estas espécies foram determinadas a partir da análise de 130 amostras de impressões de folhas de angiospermas, as quais foram coletadas durante a escavação do calcário de Caieira (Duarte, 1972).

\section{Análise da Margem Foliar}

A Análise da Margem Foliar é um método univariado para reconstruir a TMA, a qual relaciona a proporção de espécies de dicotiledôneas lenhosas sem dentes de uma flora com a TMA (Wilf, 1997; Kennedy et al., 2014). Uma vez ao que o tipo de margem foliar num conjunto florístico está submetido a restrições filogenéticas e históricas, esta correlação não é globalmente uniforme (Kennedy et al., 2014). Portanto, para obter reconstruções confiáveis, é recomendável a utilização de uma calibração no contexto de uma história fitogeográfica compartilhada (Hinojosa et al., 2011). Também se recomenda a utilização de um mínimo de 25 a 30 espécies para obter reconstruções confiáveis (Wilf, 1997; Burnham et al., 2005), embora um número inferior de espécies também as possa gerar (Ricardi-Branco et al., 2015). As reconstruções da TMA baseadas na Análise da Margem Foliar, geralmente apresentam um erro de $\pm 5^{\circ} \mathrm{C}$ e, caso se utilize uma calibração regional apropriada, o erro é reduzido para aproximadamente $\pm 2^{\circ} \mathrm{C}$ (Royer, 2012). Em geral, este método subestima a TMA (Peppe et al., 2011), uma vez que as floras fósseis associadas a depósitos fluviais ou lacustres apresentam uma grande proporção de espécies com margem com dentes (Burnham et al., 2001).

Para a reconstrução da TMA, foram selecionadas três equações derivadas de diferentes conjuntos de dados (Tabela 2). A primeira equação foi selecionada devido à sua precisão na reconstrução da TMA atual da região (Kowalski, 2002), embora seja derivada de um conjunto de dados do hemisfério norte (Wilf, 1997). A segunda e a terceira equação foram selecionadas por terem sido derivadas de conjuntos de dados da América do Sul, sendo, portanto, mais adequadas para a reconstrução da TMA de floras fósseis da região (Hinojosa et al., 2011).

\section{Análise da Área Foliar}

A Análise da Área Foliar é um método univariado para reconstruir a PMA, baseado na relação da área foliar média de uma flora com a PMA (Wilf et al., 1998). Para obter reconstruções confiáveis com base neste método, recomenda-se a utilização de um mínimo de 25 a 30 espécies para sua aplicação (Wilf et al., 1998; Jacobs \& Herendeen, 2004), embora um número inferior de espécies também as possa gerar (Ricardi-Branco et al., 2015). As reconstruções da PMA baseadas na Análise da Área Foliar, devem ser cuidadosamente interpretadas (Wilf et al., 1998; Peppe et al., 2011), já que estas podem chegar a apresentar um erro de \pm 500 $\mathrm{mm}$ (Wilf et al., 1998) a $\pm 1000 \mathrm{~mm}$ (Peppe et al., 2011). Isto se deve ao fato de que as folhas podem refletir erroneamente a PMA, devido às características da temperatura, do solo e/ ou das águas subterrâneas (Royer, 2012), assim como ao transporte que sofrem antes de sua deposição e ao ambiente (Ellis \& Johnson, 2013). Para a reconstrução da PMA, foram selecionadas três equações derivadas de diferentes conjuntos de dados (Tabela 2).

Tabela 1. Tipo de margem e tamanho das folhas de 19 espécies de angiospermas da tafoflora de Caieira, Formação Pirabas (Duarte, 1972). Símbolo: a, tamanho segundo Raunkiaer (1934) modificado por Webb (1959).

Table 1. Margin type and size of the leaves of 19 species angiosperms of the Caieira taphoflora, Pirabas Formation (Duarte, 1972). Symbol: a, size according to Raunkiaer (1934) modified by Webb (1959).

\begin{tabular}{|c|c|c|c|}
\hline Família & Espécie & Tipo de margem & Tamanho $^{\mathrm{a}}$ \\
\hline \multirow[t]{2}{*}{ Annonaceae } & Guatteria ackermannii & Sem dentes & Mesofilo \\
\hline & Guatteria basilata & Sem dentes & Mesofilo \\
\hline Bonnetiaceae & Bonnetia frequens & Sem dentes & Mesofilo \\
\hline Caryocaraceae & Caryocar recognitum & Com dentes & Mesofilo \\
\hline Chrysobalanaceae & Hirtella berryana & Sem dentes & Notofilo \\
\hline Dilleniaceae & Davilla destitute & Sem dentes & Notofilo \\
\hline Ebenaceae & Diospyros sculpata & Sem dentes & Mesofilo \\
\hline Euphorbiaceae & Drypetes capanemensis & Sem dentes & Mesofilo \\
\hline Lauraceae & Endlicheria neotropica & Sem dentes & Mesofilo \\
\hline Malvaceae & Apeiba pulchra & Sem dentes & Mesofilo \\
\hline Melastomataceae & Meriania deficiens & Sem dentes & Notofilo \\
\hline Meliaceae & Trichilia antecedens & Sem dentes & Notofilo \\
\hline Myrtaceae & Myrcia pirabensis & Sem dentes & Mesofilo \\
\hline Nyctaginaceae & Pisonia ampliata & Sem dentes & Mesofilo \\
\hline Rhizophoraceae & Cassipourea brasiliensis & Sem dentes & Mesofilo \\
\hline Rubiaceae & Faramea lapidescens & Sem dentes & Notofilo \\
\hline Rutaceae & Hortia paraensis & Sem dentes & Mesofilo \\
\hline \multirow[t]{2}{*}{ Sapindaceae } & Sapindus ferreirai & Sem dentes & Notofilo \\
\hline & Serjania decursiva & Com dentes & Microfilo \\
\hline
\end{tabular}


Tabela 2. Equações baseadas na Análise da Margem Foliar e Análise da Área Foliar utilizadas para a reconstrução da temperatura média anual (TMA) e a precipitação média anual (PMA) da tafoflora de Caieira, Formação Pirabas. Símbolos: a , número de locais; ${ }^{\text {b }}$, coeficiente de determinação; ${ }^{\text {c}}$, erro padrão do modelo; ' , região geográfica onde foram coletadas as amostras; e, proporção de espécies sem dentes; ${ }^{\text {f }}$, Log natural da área foliar calculado de acordo com Wilf et al. (1998): $M \ln A=\Sigma a_{i} p_{i}\left(a_{i}=\right.$ sete médias das áreas do log natural das classes de tamanho de Raunkiaer (1934), modificadas pelo Webb (1959), e $p_{i}=$ a proporção de espécies em cada uma das classes de tamanho).

Table 2. Equations based on the Leaf Margin Analysis and Leaf Area Analysis used to the reconstruction of the mean annual temperature (MAT) and the mean annual precipitation (MAP) of the Caieira taphoflora, Pirabas Formation. Symbols: ${ }^{\text {a }}$, number of localities; ${ }^{\text {b }}$, coefficient of determination; ${ }^{\text {c }}$, standard error of the model; ${ }^{\mathrm{d}}$, geographic region where the samples were collected; ${ }^{\mathrm{e}}$, proportion of untoothed species; ${ }^{\mathrm{f}}$, natural logarithm of the leaf área calculated according to Wilf et al. (1998): $M \ln A=\Sigma a p_{i}\left(a_{i}=\right.$ seven means of the areas of the natural logarithm of the Raunkiaer (1934) size classes, modified by Webb (1959), and $p_{i}=$ the proportion of species in each size class).

\begin{tabular}{|c|c|c|c|c|c|c|}
\hline & Equação & $\mathrm{n}^{\mathrm{a}}$ & $\mathrm{r}^{2 \mathrm{~b}}$ & $\mathrm{EP}^{\mathrm{c}}$ & Região $^{d}$ & Fonte \\
\hline \multirow{3}{*}{$\begin{array}{l}\text { Análise da } \\
\text { Margem Foliar }\end{array}$} & $\begin{array}{c}(\text { Eq. } 1) \\
\mathrm{TMA}=24,40 E^{\mathrm{e}}+3,25\end{array}$ & 74 & 0,84 & 2,1 & $\begin{array}{c}\text { América do Norte } \\
\text { e América Central e Japão }\end{array}$ & Wilf (1997) \\
\hline & $\begin{array}{c}(\text { Eq. } 2) \\
\mathrm{TMA}=23,42 E+3,60\end{array}$ & 44 & 0,48 & 3,5 & $\begin{array}{l}\text { Zona tropical da América do Sul } \\
\text { (Bolívia, Brasil, Colômbia, Equador, } \\
\text { Guiana, Peru e Venezuela) }\end{array}$ & $\begin{array}{l}\text { Hinojosa et al. } \\
\qquad(2011)\end{array}$ \\
\hline & $\begin{array}{c}(\text { Eq. } 3) \\
\mathrm{TMA}=26,03 E+1,31\end{array}$ & 74 & 0,82 & 2,8 & $\begin{array}{c}\text { América do Sul (Bolívia, Brasil, } \\
\text { Colômbia, Chile, Equador, Guiana, } \\
\text { Peru e Venezuela) }\end{array}$ & $\begin{array}{l}\text { Hinojosa et al. } \\
\text { (2011) }\end{array}$ \\
\hline \multirow{3}{*}{$\begin{array}{l}\text { Análise da } \\
\text { Área Foliar }\end{array}$} & $\begin{array}{c}\text { (Eq. 4) } \\
\ln \mathrm{PMA}=0,548 M \ln A^{\mathrm{f}}+0,768\end{array}$ & 50 & 0,760 & 0,359 & $\begin{array}{c}\text { América do Norte, América Central, } \\
\text { América do Sul e África }\end{array}$ & $\begin{array}{l}\text { Wilf et al. } \\
\text { (1998) }\end{array}$ \\
\hline & $\begin{array}{c}\text { (Eq. 5) } \\
\ln \mathrm{PMA}=2,566+0,309 \mathrm{M} \ln A\end{array}$ & 42 & 0,734 & -------- & Zona tropical da África e Bolívia & $\begin{array}{c}\text { Jacobs \& Herendeen } \\
\text { (2004) }\end{array}$ \\
\hline & $\begin{array}{c}\text { (Eq. 6) } \\
\ln \mathrm{PMA}=2,167+0,354 M \ln A\end{array}$ & 79 & 0,709 & -------- & $\begin{array}{c}\text { América do Norte, América Central, } \\
\text { América do Sul e África }\end{array}$ & $\begin{array}{c}\text { Jacobs \& Herendeen } \\
\text { (2004) }\end{array}$ \\
\hline
\end{tabular}

\section{RESULTADOS}

Das 19 espécies analisadas da tafoflora de Caieira, 17 apresentam margens sem dentes, o que equivale ao $89,5 \%$ do total, valor este que permite reconstruir a TMA (Tabela $3)$. As equações selecionadas produziram diferentes valores que vão de 24,6 a $25,0^{\circ} \mathrm{C}$ (Tabela 3). A partir dos dados

Tabela 3. Reconstrução da temperatura média anual (TMA) e a precipitação média anual (PMA) da tafoflora de Caieira, Formação Pirabas, com base na Análise da Margem Foliar e na Análise da Área Foliar. Símbolos: a , proporção de espécies sem dentes; ${ }^{\text {b }}$, logaritmo natural da área foliar; ${ }^{\mathrm{c}}$, temperatura média anual e precipitação média anual do Município de Capanema (https:// pt.climate-data.org/).

Table 3. Reconstruction of the mean annual temperature (MAT) and the mean annual precipitation (MAP) of the Caieira taphoflora, Pirabas Formation, based on the Leaf Margin Analysis and Leaf Area Analysis. Symbols: ${ }^{\text {a }}$, proportion of untoothed species; ${ }^{b}$, natural logarithm of leaf area; ${ }^{\mathrm{c}}$, mean annual temperature and mean annual precipitation of the Municipality of Capanema (https://pt.climate-data.org/).

\begin{tabular}{cccc}
\hline \multicolumn{2}{c}{$\begin{array}{c}\text { Análise da Margem Foliar } \\
E=0,895^{\mathrm{a}}\end{array}$} & \multicolumn{2}{c}{$\begin{array}{c}\text { Análise da Área Foliar } \\
M \ln A=8,63^{\mathrm{b}}\end{array}$} \\
\hline Equação & TMA $\left({ }^{\circ} \mathrm{C}\right)$ & Equação & PMA (mm) \\
\hline Eq. 1 & $25,0 \pm 2,1$ & Eq. 4 & $2423^{+1040}-731$ \\
Eq. 2 & $24,6 \pm 3,5$ & Eq. 5 & 1868 -------- \\
Eq. 3 & $24,6 \pm 2,8$ & Eq. 6 & 1849 -------- \\
\hline Atual $^{\mathrm{c}}$ & 26,1 & Atual & 2468 \\
\hline
\end{tabular}

da área foliar das 19 espécies analisadas determinou-se inicialmente a proporção relativa em cada classe de tamanho e posteriormente o logaritmo natural da área foliar (Tabela 4). Este valor permite reconstruir a PMA (Tabela 3). As equações selecionadas produziram diferentes valores que vão de 1849 a $2423 \mathrm{~mm}$ (Tabela 3).

Tabela 4. Dados da área foliar das folhas de 19 espécies de angiospermas da tafoflora de Caieira, Formação Pirabas. Símbolos: a , classes de tamanho segundo Raunkiaer (1934) modificadas por Webb (1959); ' , proporções relativas; ${ }^{\mathrm{c}}$, média da área log natural para cada classe de tamanho; ${ }^{\mathrm{d}}, \log$ natural da área foliar calculado de acordo com Wilf et al. (1998), onde $M \ln A=\Sigma a_{i} p_{i}$.

Table 4. Leaf area data of the leaves of 19 species angiosperms of the Caieira taphoflora, Pirabas Formation. Symbols: a, size classes according to Raunkiaer (1934) modified by Webb (1959); ${ }^{\mathbf{b}}$, relative proportions; ${ }^{\mathbf{c}}$, mean of the natural logarithm area for each size class; ${ }^{\mathrm{d}}$, natural logarithm of the leaf area calculated according to Wilf et al. (1998), where $M \ln A=\Sigma a p_{i}$.

\begin{tabular}{|c|c|c|c|c|}
\hline $\begin{array}{l}\text { Classe de } \\
\text { tamanho }^{\mathrm{a}}\end{array}$ & $\begin{array}{l}\text { Faixa de classe de } \\
\text { tamanho }\left(\mathrm{cm}^{2}\right)\end{array}$ & $\begin{array}{c}p_{i} \text { Classes de } \\
\text { tamanho }^{\mathrm{b}}\end{array}$ & $a_{i}^{\mathrm{c}}$ & $M \ln A^{\mathrm{d}}\left(a_{i} p_{i}\right)$ \\
\hline Leptofilo & $\leq 0,25$ & 0,00 & 2,12 & 0,00 \\
\hline Nanofilo & $>0,25-2,25$ & 0,00 & 4,32 & 0,33 \\
\hline Microfilo & $2,25-20,25$ & 0,05 & 6,51 & 2,56 \\
\hline Notofilo & $20,25-45,00$ & 0,32 & 8,01 & 5,74 \\
\hline Mesofilo & $45,00-182,25$ & 0,63 & 9,11 & 0,00 \\
\hline Macrofilo & $182,25-1640,20$ & 0,00 & 10,90 & 0,00 \\
\hline \multirow[t]{2}{*}{ Megafilo } & $>1640,20$ & 0,00 & 13,10 & 0,00 \\
\hline & & & $\Sigma a p_{i}$ & 8,63 \\
\hline
\end{tabular}




\section{DISCUSSÃO E CONCLUSÕES}

A reconstrução do clima do Cenozoico da Amazônia com base em floras fósseis se vê afetada pela contínua elevação dos Andes e as mudanças climáticas (Kowalski, 2002), já que estes fatores originaram mudanças ambientais em escala regional e local, controlando a distribuição da vegetação (Rossetti \& Toledo, 2007). Apesar disto, as 19 espécies da tafoflora de Caieira analisadas permitiram a reconstrução da TMA e a PMA para o momento de sua deposição (Tabela 3).

A vegetação atual da Amazônia remonta ao início do Paleógeno (Rossetti \& Toledo, 2007; Jaramillo et al., 2010), mas ficou firmemente estabelecida a partir do Mioceno (Mörner, 2016). Contudo, em determinadas áreas, como a de ocorrência dos depósitos da Formação Pirabas, a vegetação teria se estabelecido no Oligoceno/Mioceno (Hoorn et al., 2010; Jaramillo et al., 2010). Isto é demonstrado pela semelhança que apresenta a vegetação natural para o momento da deposição da Formação Pirabas com a atual vegetação natural da área de ocorrência dos depósitos da Formação Pirabas. Para o momento da deposição da Formação Pirabas, a vegetação natural estava composta de floresta de terra firme, floresta de planície inundável, floresta de pântano, restinga e manguezal (Duarte, 1972, 2004; Leite et al., 1997; Leite, 1997, 2004; Antonioli et al., 2015). Esta vegetação foi similar à vegetação natural atual da área de ocorrência da Formação Pirabas, a qual está composta por floresta de planície inundável, restinga, manguezal e campos salinos (IBGE, 2008).

Assim como a vegetação da área de ocorrência da Formação Pirabas, a atual configuração climática da Amazônia também remonta ao Oligoceno/Mioceno (Jaramillo, 2012; Hoorn et al., 2014), mas desde o Mioceno é que se tornou estável, exceto para algumas pequenas áreas da periferia (Colinvaux et al., 2001).

A área de ocorrência da Formação Pirabas encontra-se atualmente sob um clima quente e úmido (IBGE, 2002), produto da influência da Zona de Convergência Intertropical, da Zona de Convergência do Atlântico Sul, das Linhas de Instabilidade e dos sistemas frontais advindos do Sul/Sudeste do Brasil (Amanajás \& Braga, 2012; Souza \& Rocha, 2014). Este clima é similar aquele sob qual esteve submetido a tafoflora de Caieira, assim como toda a área de ocorrência da Formação Pirabas (Duarte, 1972, 2004; Leite, 1997, 2004; Leite et al., 1997; Jaramillo et al., 2010; Hoorn et al., 2014; Távora et al., 2014; Antonioli et al., 2015), e toda a Amazônia durante o Oligoceno/Mioceno (Hoorn et al., 2010, 2014).

O clima atual da área de ocorrência da Formação Pirabas se caracteriza por apresentar uma TMA de 25 a $26^{\circ} \mathrm{C}$ e uma PMA de 2000 a 3000 mm (SUDAM/PHCA, 1984; Oliveira et al., 2004). Estes valores de TMA são similares aos obtidos para a tafoflora de Caieira (Tabela 3). Em contrapartida, os valores de PMA são superiores a alguns dos obtidos (Tabela 3), devido ao fato da tafoflora de Caieira não estar reproduzindo o valor real da PMA, já que o processo de fossilização não permitiu a conservação de folhas com maior área foliar, especificamente as megáfilas (Duarte, 1972, 2004). Apesar disto, é possível inferir que a atual configuração climática da área de ocorrência da Formação Pirabas tem permanecido estável desde o Oligoceno/Mioceno, assim como sua vegetação.

Atualmente a região onde se encontrava a tafoflora de Caieira apresenta uma TMA de $26,1^{\circ} \mathrm{C}$ e uma PMA de 2468 $\mathrm{mm}$ (Tabela 3). Ao comparar estes valores com os obtidos para a tafoflora de Caieira, observa-se que para o momento de sua deposição as condições eram mais frias, em 1,1 a $1,5^{\circ} \mathrm{C}$, e menos úmidas, em 42 a 619 mm (Tabela 3). Possivelmente, isto seja resultado do evento de arrefecimento global que afetou de maneira geral a Amazônia durante a transição Oligoceno/Mioceno (Stewart et al., 2016). Este evento, conhecido como glaciação Mi-1, caracterizou-se por uma grande expansão da camada de gelo da Antártida (Zachos et al., 2001; Lear et al., 2004), e pela diminuição de $2^{\circ} \mathrm{C}$ na temperatura do oceano profundo (Lear et al., 2004).

\section{AGRADECIMENTOS}

Os autores agradecem o apoio financeiro da Fundação de Amparo à Pesquisa do Estado de São Paulo (FAPESP; projeto Studies of Modern and fossil bioclast accumulations related to continental and coastal environments - 2016/209270 ), à Coordenação de Aperfeiçoamento de Pessoal de Nível Superior (CAPES) e ao Conselho Nacional de Desenvolvimento Científico e Tecnológico (CNPq) pelas bolsas concedidas. Os autores também expressam seus agradecimentos aos avaliadores anônimos e ao editor, A.M. Ribeiro, pelas valiosas sugestões que ajudaram a melhorar o manuscrito.

\section{REFERÊNCIAS}

Ackerman, F.L. 1964. Geologia e fisiografia da Região Bragantina (Estado do Pará). Cadernos da Amazônia, 2:1-90.

Aguilera, O.; Guimarães, J.T.F. \& Moraes-Santos, H. 2014. Erratum to: Neogene eastern Amazon carbonate platform and the paleoenvironmental interpretation. Swiss Journal of Palaeontology, 133:99-118. doi:10.1007/s13358-014-0066-6

Amanajás, J.C. \& Braga, C.C. 2012. Padrões espaço-temporal pluviométricos na Amazônia oriental utilizando análise multivariada. Revista Brasileira de Meteorologia, 27:423-434. doi:10.1590/S0102-77862012000400006

Antonioli, L.; Tavora, V.A. \& Dino, R. 2015. Palynology of carcinolites and limestones from the Baunilha Grande ecofacies of the Pirabas Formation (Miocene of Pará state, northeastern Brazil). Journal of South American Earth Sciences, 62:134-147. doi:10.1016/j.jsames.2015.05.005

Burnham, R.J.; Ellis, B. \& Johnson, K.R. 2005. Modern tropical forest taphonomy: does high biodiversity affect paleoclimatic interpretations? Palaios, 20:439-451. doi:10.2110/palo.2004. P04-60

Burnham, R.J.; Pitman, N.C.A.; Johnson, K.R. \& Wilf, P. 2001. Habitat-related error estimating temperatures from leaf margins 
in a humid tropical forest. American Journal of Botany, 88:10961102. doi:10.2307/2657093

Burnham, R.J. \& Tonkovich, G.S. 2011. Climate, leaves, and the legacy of two giants. New Phytologist, 190:514-517. doi:10.1111/j.1469-8137.2011.03725.x

Colinvaux, P.A.; Irion, G.; Räsänen, M.E.; Bush, M.B. \& Mello, J.A.S.N. 2001. A paradigm to be discarded: geological and paleoecological data falsify the Haffer \& Prance refuge hypothesis of Amazonian speciation. Amazoniana, 16:609-646.

Duarte, L. 1972. Flórula da Formação Pirabas, Estado do Pará, Brasil. Programa de Pós-graduação em Paleontologia e Estratigrafia, Universidade de São Paulo, Tese de Doutorado, $181 \mathrm{p}$.

Duarte, L. 2004. Paleoflórula. In: D.F. Rossetti \& A.M. Góes, (eds.) O Neógeno da Amazônia Oriental, Museu Paraense Emílio Goeldi, p. 169-198.

Ellis, B. \& Johnson, K.R. 2013. Comparison of leaf samples from mapped tropical and temperate forests: implications for interpretations of the diversity of fossil assemblages. Palaios, 28:163-177. doi:10.2110/palo.2012.p12-073r

Hinojosa, L.F.; Pérez, F.; Gaxiola, A. \& Sandoval, I. 2011. Historical and phylogenetic constraints on the incidence of entire leaf margins: insights from a new South American model. Global Ecology and Biogeography, 20:380-390. doi:10.1111/j.14668238.2010.00595.x

Hoorn, C.; Bernardes-de-Oliveira, M.E.C.; Dino, R.; Garcia, M.J.; Antonioli, L.; Casado, F.C. \& Hooghiemstra, H. 2014. Neogene climate evolution in Amazonia and Brazilian Northeast. In: I.S. Carvalho; M.J. Garcia; C.C. Lana \& O. Strohschoen Jr. (eds.) Paleontologia: Cenários de Vida-Paleoclimas, Interciência, p. 277-310.

Hoorn, C; Wesselingh, F.P.; Hovikoski, J. \& Guerrero, J. 2010. The development of the Amazonian mega-wetland (Miocene; Brazil, Colombia, Peru, Bolivia). In: C. Hoorn \& F.P. Wesselingh (eds.) Amazonia, landscape and species evolution: a look into the past, Blackwell Publishing, p. 123-142. doi:10.1002/9781444306408. ch8

IBGE (Instituto Brasileiro de Geografia e Estatística). 2002. Mapa de Clima do Brasil. Escala 1:500.000. Disponível em: ftp://geoftp. ibge.gov.br/informacoes_ambientais/climatologia/mapas/brasil/ clima.pdf; acesso em 13/08/2017.

IBGE (Instituto Brasileiro de Geografia e Estatística). 2008. Estado do Pará. Vegetação. Escala 1:1.800.000. Disponível em: ftp:// geoftp.ibge.gov.br/informacoes_ambientais/vegetacao/mapas/ unidades_da_federa $\% \mathrm{C} 3 \% \mathrm{~A} 7 \% \mathrm{C} 3 \% \mathrm{~A} 3 \mathrm{o} / \mathrm{pa}$ _vegetacao.pdf; acesso em 13/08/2017.

Jacobs, B.F. \& Herendeen, P.S. 2004. Eocene dry climated and woodland vegetation in tropical Africa reconstructed from fossil leaves from northern Tanzania. Palaeogeography, Palaeoclimatology, Palaeoecology, 213:115-123. doi:10.1016/j. palaeo.2004.07.007

Jaramillo, C.A. 2012. Historia geológica del Bosque Húmedo Tropical. Revista de la Academia Colombiana de Ciencias Exactas, Físicas y Naturales, 36:57-80.

Jaramillo, J.; Hoorn, C.; Silva, S.A.F.; Leite, F.; Herrera, F.; Quiroz, L.; Dino, R. \& Antonioli, L. 2010. The origin of the modern Amazon rainforest: implications of the palynological and palaeobotanical record. In: C. Hoorn \& F.P. Wesselingh (eds.) Amazonia, landscape and species evolution: a look into the past, Blackwell Publishing, p. 317-334. doi:10.1002/9781444306408.ch19
Kennedy, E.M.; Arens, N.C.; Reichgelt, T.; Spicer, R.A.; Spicer, T.E.V.; Stranks, L. \& Yang, J. 2014. Deriving temperature estimates from Southern Hemisphere leaves. Palaeogeography, Palaeoclimatology, Palaeoecology, 412:80-90. doi:10.1016/j. palaeo.2014.07.015

Kowalski, E.A. 2002. Mean annual temperature estimation based on leaf morphology: a test from tropical South America. Palaeogeography, Palaeoclimatology, Palaeoecology, 188:141165. doi:10.1016/S0031-0182(02)00550-3

Lear, C.H.; Rosenthal, Y.; Coxall, H.K. \& Wilson, P.A. 2004. Late Eocene to early Miocene ice sheet dynamics and the global carbon cycle. Paleoceanography, 19:PA4015. doi:10.1029/2004PA001039

Leite, F.P.R. 1997. Palinofloras neógenas da Formação Pirabas e Grupo Barreiras, área litorânea nordeste do estado do Pará, Brasil. Programa de Pós-graduação em Geologia Sedimentar, Universidade de São Paulo, Dissertação de Mestrado, 102 p.

Leite, F.P.R. 2004. Palinologia. In: D.F. Rossetti \& A.M. Góes (eds.) O Neógeno da Amazônia Oriental, Museu Paraense Emílio Goeldi, p. 55-90.

Leite, F.P.R.; Bernardes-de-Oliveira, M.E.C.; Oliveira, P.E.; Silvestre-Capelato, M.S.; Arai, M. \& Truckenbrodt, W. 1997. Palinofloras miocenas da Formação Pirabas e Grupo Barreiras na região Bragantina, Estado do Pará Brasil. Revista Universidade Guarulhos, Geociências, 2:128-140.

Mörner, N.-A. 2016. Origin of the Amazonian rainforest. International Journal of Geosciences, 7:470-478. doi:10.4236/ ijg.2016.74036

Oliveira, L.L.; Fontinhas, R.L.; Lima, A.M.M. \& Lima, R.J.S. 2004. Mapas dos parâmetros climatológicos do estado do Pará: umidade, temperatura e insolação, médias anuais. In: CONGRESSO BRASILEIRO DE METEREOLOGIA, 13, 2004. Resumos, Fortaleza, SBM, p. 7.

Peppe, D.J. et al. 2011. Sensitivity of leaf size and shape to climate: global patterns and paleoclimatic applications. New Phytologist, 190:724-739. doi:10.1111/j.1469-8137.2010.03615.x

Raunkiaer, C. 1934. The life forms of plants and statistical plant geography. Oxford, Clarendon Press, $632 \mathrm{p}$.

Ricardi-Branco, F.; Pereira, S.Y.; Souza, M.M.; Santiago, F.; Pereira, P.B.P.; Branco, F.C.; Ribeiro, V. \& Molina, K. 2015. Relationships among subaquatic environment and leaf/ palinomorph assemblages of the Quaternary Mogi-Guaçú River alluvial plain, SP, Brazil. In: M. Ramkumar; K. Kumaraswamy \& R. Mohanraj (eds.) Environmental management of river basin ecosystems, Springer, p. 667-705. doi:10.1007/978-3319-13425-3 30

Rossetti, D.F. \& Góes, A.M. 2004. Geologia. In: D.F. Rossetti \& A.M. Góes (eds.) O Neógeno da Amazônia Oriental, Museu Paraense Emílio Goeldi, p. 13-52.

Rossetti, D.F. \& Toledo, P.M. 2007. Environmental changes in Amazonia as evidenced by geological and paleontological data. Revista Brasileira de Ornitologia, 15:251-264.

Royer, D.L. 2012. Climate reconstruction from leaf size and shape: new developments and challenges. In: L.C. Ivany \& B.T. Huber (eds.) Reconstructing earth's deep-time climate, Bethesda, The Paleontological Society, p. 195-212 (Papers 18).

Silva, C.B. 2016. Palinologia da Formação Pirabas, nos municípios de Primavera e Salinópolis, nordeste do estado do Pará, Brasil. Programa de Pós-graduação em Geologia e Geoquímica, Universidade Federal do Pará, Dissertação de Mestrado, 222 p. 
Souza, E.B. \& Rocha, J.P. 2014. Climatologia, variabilidade e tendências do clima atual na Amazônia e em cenários futuros de mudanças climáticas. In: I.C.G Vieira; P.M. Toledo \& R.A.O. Santos Jr. (eds.) Ambiente e sociedade na Amazônia: uma abordagem interdisciplinar, Garamond, p. 295-312.

Stewart, J.A.; Gutjahr, M.; James, R.H.; Anand, P. \& Wilson, P.A. 2016. Influence of the Amazon River on the $\mathrm{Nd}$ isotope composition of deep water in the western equatorial Atlantic during the Oligocene-Miocene transition. Earth and Planetary Science Letters, 454:132-141. doi:10.1016/j.epsl.2016.08.037

SUDAM/PHCA (Superintendência do desenvolvimento da Amazônia/Projeto de Hidrologia e Climatologia da Amazônia). 1984. Atlas climatológico da Amazônia brasileira. Belém, Superintendência do desenvolvimento da Amazônia, 125 p.

Távora, V.A.; Rodrigues, L.F.F. \& Cunha, T.S. 2014. Paleoclima da Amazônia oriental durante o Mioceno. In: I.S. Carvalho; M.J. Garcia; C.C. Lana \& O. Strohschoen Jr. (eds.) Paleontologia: Cenários de Vida-Paleoclimas, Interciência, p. 311-316.

Távora, V.A.; Santos, A.A.R. \& Araújo, R.N. 2010a. Eventos biológicos da Formação Pirabas (Mioceno Inferior). Revista Brasileira de Geociências, 40:256-264.
Távora, V.A.; Santos, A.A.R. \& Araújo, R.N. 2010b. Localidades fossilíferas da Formação Pirabas (Mioceno Inferior). Boletim do Museu Paraense Emílio Goeldi, Ciências Naturais, 5:207-224.

Webb, L.J. 1959. A physiognomic classification of Australian rain forest. Journal of Ecology, 47:551-570. doi:10.2307/2257290

Wilf, P. 1997. When are leaves good thermometers? A new case for leaf margin analysis. Paleobiology, 23:373-390. doi:10.1017/ S0094837300019746

Wilf, P.; Wing, S.L.; Greenwood, D.R. \& Greenwood, C.L. 1998. Using fossil leaves as paleoprecipitation indicators: an Eocene example. Geology, 26:203-206. doi:10.1130/00917613(1998)026<0203:UFLAPI >2.3.CO;2

Wright, I.J. et al. 2017. Global climatic drivers of leaf size. Science, 357:917-921. doi:10.1126/science.aal4760

Zachos, J.C.; Pagani, M.; Sloan, L.; Thomas, E. \& Billups, K. 2001. Trends, rhythms, and aberrations in global climate $65 \mathrm{Ma}$ to present. Science, 292:686-693. doi:10.1126/science. 1059412

Received in 05 April, 2018; Accepted in 02 September, 2018. 